\title{
Épidémie de COVID-19 chez les travailleurs étrangers temporaires en Colombie-Britannique, entre mars et mai 2020
}

\author{
Silvina Mema ${ }^{1 *}$, Gillian Frosst ${ }^{1}$, Kristen Hanson ${ }^{1}$, Cheryl Yates ${ }^{1}$, Amanda Anderson ${ }^{1}$, \\ Jennifer Jacobsen ${ }^{1}$, Caroline Guinard ${ }^{2}$, America Lima ${ }^{3}$, Tannis Andersen ${ }^{1}$, Melissa Roe ${ }^{1}$
}

\section{Résumé}

Contexte : Pendant la pandémie de la maladie à coronavirus 2019 (COVID-19), les travailleurs étrangers temporaires ont joué un rôle essentiel dans le maintien de l'approvisionnement alimentaire au Canada, mais ils ont également dû relever un certain nombre de défis qui les ont rendus particulièrement vulnérables à la COVID-19. Cette étude vise à décrire l'enquête épidémiologique et la réponse de la santé publique à une éclosion de COVID-19 chez les travailleurs étrangers temporaires dans un cadre agricole en Colombie-Britannique qui s'est produite entre mars et mai 2020.

Méthodes : L'éclosion a été déclarée le 28 mars 2020 à la suite de la détection de deux cas de COVID-19 chez un groupe de 63 travailleurs étrangers temporaires employés dans une pépinière et un centre de jardinage. Les mesures de lutte contre les éclosions ont inclus I'isolement immédiat des personnes atteintes, la recherche de cas par le dépistage et les tests de sensibilisation, la mise en cohorte des travailleurs asymptomatiques et l'amélioration du nettoyage et de la désinfection. L'éclosion a été déclarée terminée le 10 mai 2020.

Résultats : Au total, 26 cas de COVID-19 ont été détectés dans le groupe des travailleurs étrangers temporaires, mais aucun cas n'a été décelé parmi les travailleurs locaux. Les personnes atteintes étaient principalement de sexe masculin $(77 \%)$ avec un âge médian de 41 ans. Les symptômes se sont manifestés entre le 8 mars et le 9 avril 2020. Un cas a nécessité une hospitalisation d'une nuit en raison d'une pneumonie.

Conclusion : II s'agissait de la première éclosion de COVID-19 en Colombie-Britannique et de la première éclosion de COVID-19 chez les travailleurs étrangers temporaires au Canada. Cette éclosion a commencé avant la mise en œuvre des ordonnances de quarantaine provinciales et fédérales pour les voyageurs internationaux. Une politique provinciale qui exige que les travailleurs étrangers temporaires soient mis en quarantaine dans des locaux financés par le gouvernement avant d'être déployés dans des milieux agricoles a ensuite été élaborée.
Cette oeuvre est mise à la disposition selon les termes de la licence internationale Creative Commons Attribution 4.0

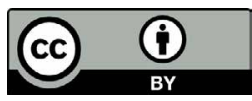

Affiliations

${ }^{1}$ Interior Health, Kelowna, BC

2 Faculté de médecine, Université de Colombie-Britannique, Vancouver, BC

${ }^{3}$ Médecin de famille à la clinique de Turtle Lake, Lake Country, BC

${ }^{4}$ Laboratoire de santé publique du Centre de contrôle des maladies de la ColombieBritannique, Vancouver, BC

\section{*Correspondance :}

silvina.mema@interiorhealth.ca
Citation proposée : Mema SC, Frosst G, Hanson K, Yates C, Anderson A, Jacobsen J, Guinard C, Lima AU,

Andersen T, Roe M. Épidémie de COVID-19 chez les travailleurs étrangers temporaires en Colombie-Britannique, entre mars et mai 2020. Relevé des maladies transmissibles au Canada 2021;47(1):6-12.

https://doi.org/10.14745/ccdr.v47i01a02f

Mots-clés : COVID-19, éclosions, travailleurs étrangers temporaires, travailleurs migrants, travailleurs agricoles

\section{Introduction}

En décembre 2019, le nouveau coronavirus du syndrome respiratoire aigu sévère 2 (SRAS-CoV-2) est apparu à Wuhan, en Chine, avant de se répandre partout dans le monde. La maladie du coronavirus de 2019 (COVID-19) se manifeste habituellement comme un syndrome grippal et le virus est principalement transmis entre les personnes par des gouttelettes respiratoires et utilise différentes voies de contact qui entraînent des éclosions à grande échelle. En réponse à la propagation rapide et à la nécessité d'une intervention internationale coordonnée, l'Organisation mondiale de la Santé (OMS) a déclaré l'éclosion de la COVID-19 comme une urgence sanitaire mondiale le 30 janvier 2020, puis comme une pandémie le 11 mars 2020 (1). 
Les voyageurs internationaux sont plus à risque de contracter la COVID-19 et de contribuer à sa dissémination (2). C'est pour cette raison que les gouvernements du monde entier ont imposé des restrictions strictes concernant les voyages internationaux afin de limiter les répercussions mondiales du virus. Au Canada, certaines catégories de personnes qui fournissaient des services essentiels étaient exemptées des restrictions relatives aux déplacements, ce qui incluait les travailleurs étrangers temporaires qui jouent un rôle essentiel pendant la saison des récoltes en contribuant à l'économie et en maintenant l'approvisionnement alimentaire $(3,4)$. Les travailleurs étrangers temporaires sont des travailleurs migrants qui viennent chaque année au Canada, généralement d'Amérique latine, pour travailler dans le secteur agricole. La vallée de l'Okanagan en Colombie-Britannique (C.-B.), au Canada, est un important centre de production agricole et le fonctionnement de l'industrie agricole dans cette région dépend des travailleurs étrangers temporaires.

Au début de la pandémie, soit entre mars et mai 2020, une éclosion de COVID-19 s'est déclarée chez des travailleurs étrangers temporaires dans un établissement agricole de la région de l'Okanagan. Cette éclosion a permis d'obtenir un aperçu des éléments qui ont rendu ces travailleurs particulièrement vulnérables pendant la pandémie de COVID-19. Ces éléments peuvent inclure des obstacles linguistiques et culturels, la crainte de perdre son emploi ou même d'être expulsé si les symptômes sont divulgués, sans oublier la variabilité des conditions de vie, y compris l'accès aux vêtements et à la nourriture, la disponibilité des salaires pendant l'isolement ou la maladie, l'accès aux services médicaux et la couverture des frais médicaux, ainsi que l'accès au transport (5).

Ce rapport décrit l'enquête épidémiologique et les mesures de contrôle de santé publique mises en œuvre en réponse à l'éclosion.

\section{Méthodes}

\section{Équipe chargée de l'enquête sur l'éclosion}

L'enquête sur l'éclosion a été dirigée par le médecin-hygiéniste d'Interior Health et une équipe formée d'un épidémiologiste, de deux spécialistes des maladies transmissibles ayant de I'expérience dans la recherche des contacts, du personnel infirmier de soins primaires, d'une infirmière praticienne, de deux agents $d$ 'hygiène du milieu et $d$ 'un administrateur de services de santé. Un coordonnateur du programme des travailleurs saisonniers employés par l'entreprise agricole touchée par l'éclosion, un travailleur des services d'approche pour les migrants venus d'un centre local de ressources communautaires et deux médecins de première ligne ont fourni un soutien supplémentaire.

\section{Détection de l'éclosion et recherche de cas} Du 24 au 27 mars 2020, deux cas confirmés de COVID-19 ont été signalés dans une cohorte de 63 travailleurs étrangers temporaires employés par une pépinière et un centre de jardinage dans la région de l'Okanagan. Une éclosion a été déclarée le 28 mars 2020, suivi d'une autre qui fut déclarée le 10 mai 2020, soit 28 jours (deux périodes d'incubation) après la date où un test a été effectué chez la dernière personne atteinte.

Dans le cadre de cette enquête, les cas confirmés d'éclosion ont été définis comme des personnes qui travaillaient à la pépinière et présentaient les conditions suivantes :

- Confirmation en laboratoire du diagnostic de COVID-19 (6)

- Date d'apparition des symptômes : le $1^{\text {er }}$ mars 2020 ou à une date ultérieure

Les cas épidémiologiquement liés ont été définis comme des personnes qui travaillaient à la pépinière et présentaient les conditions suivantes :

- Symptômes compatibles avec la COVID-19

- Apparition des symptômes : le $1^{\mathrm{er}}$ mars 2020 ou par la suite

- Lien épidémiologique connu avec un cas confirmé

Les activités de recherche de cas comprenaient la vérification quotidienne des symptômes de tous les travailleurs étrangers temporaires et un questionnaire détaillé de dépistage des symptômes (voir matériel supplémentaire) auquel les travailleurs ont dû répondre à quatre reprises pendant l'enquête. Des équipes médicales de proximité ont fait passer les questionnaires en personne. Les sources de données supplémentaires comprenaient les dossiers médicaux des personnes atteintes et les entrevues effectuées dans le cadre du suivi des cas et des recherches de contacts en santé publique. Puisque les travailleurs étrangers temporaires étaient hispanophones, l'information et les services étaient fournis en espagnol dans la mesure du possible. Des services de traduction ont été fournis par I'entremise du Provincial Language Service (7), du coordonnateur du programme des travailleurs saisonniers, d'un travailleur des services d'approche pour les migrants et d'un médecin de première ligne qui parlaient tous couramment l'anglais et l'espagnol.

Tous les travailleurs qui ont signalé des symptômes compatibles avec la COVID-19 ont été référés pour un test de dépistage. Un petit nombre de travailleurs asymptomatiques jugés comme étant ceux qui présentaient le risque le plus élevé d'infection par la COVID-19 ont également subi un test de dépistage. Des spécimens respiratoires prélevés avec des écouvillons nasopharyngés ont ensuite été soumis à des essais d'amplification des acides nucléiques au laboratoire régional de microbiologie. L'extraction a été effectuée sur le système de traitement des liquides STARlet (Hamilton) à l'aide d'ensembles de cartouches universelles STARmag 96. Les tests de réaction en chaîne de la polymérase (PCR) ont été effectués à l'aide du 
CFX96 (Biorad), à l'aide de l'essai Allplex 2019-nCoV ciblant les gènes $E$, RdRP et $N$ conformément aux instructions du fabricant (Seegene).

\section{Analyse}

Panorama, le système d'information sur la santé publique d'Interior Health, a fourni les renseignements déclarables sur les cas (8). Une liste supplémentaire des 63 travailleurs étrangers temporaires a été tenue à jour et incluait des renseignements détaillés sur l'âge, le sexe, la date d'arrivée au Canada, le ou les lieux d'hébergement locaux, les tâches particulières qu'ils effectuaient à la pépinière et les dates de tests pour la COVID-19, ainsi que les résultats. Les analyses descriptives ont été effectuées à l'aide de Microsoft Excel 2010 et du logiciel SAS version 9.4.

\section{Mesures de santé publique}

Le jour où l'éclosion a été déclarée, deux agents d'hygiène du milieu ont inspecté les lieux afin d'évaluer le milieu de travail et les conditions de vie des travailleurs. Une ordonnance de la santé publique en vertu de la Public Health Act (9) de la Colombie-Britannique a été émise à l'intention de l'exploitant de l'entreprise pour lui demander d'améliorer le nettoyage des installations auxquelles ont accès les employés, d'effectuer des tests de dépistage auprès du personnel, des entrepreneurs et des visiteurs, de déclarer obligatoirement toute nouvelle maladie respiratoire chez les employés ou les entrepreneurs et de mettre en quarantaine tous les travailleurs étrangers temporaires. Cette ordonnance a ensuite été annulée, une fois l'éclosion terminée.

La gestion des cas a inclus la surveillance quotidienne conformément aux lignes directrices provisoires de la ColombieBritannique sur la gestion des cas et des contacts associés à la COVID-19 (10). Le coordonnateur du Programme des travailleurs saisonniers a également demandé des rapports quotidiens à l'Unité des maladies transmissibles d'Interior Health, qui comprenaient l'état de santé des cas et des contacts. Toute nouvelle personne symptomatique a également été signalée à l'Unité des maladies transmissibles à des fins de suivi par la santé publique. Un protocole a été établi pour transporter en toute sécurité les personnes ayant besoin de soins de santé vers des établissements de soins et en provenance de ces endroits lorsque l'utilisation d'une ambulance n'était pas indiquée. Tous les travailleurs avaient aussi accès à des rendez-vous virtuels avec un médecin de première ligne, au besoin.

Les travailleurs étrangers temporaires vivaient dans un logement partagé fourni par l'employeur, comprenant cinq maisons et 11 roulottes réparties sur cinq sites géographiquement distincts. Les personnes symptomatiques ont immédiatement été isolées, d'abord dans des logements existants. Puis, en raison du nombre croissant de cas et de la disponibilité limitée de locaux autonomes pour une seule personne, les travailleurs symptomatiques ont par la suite été relocalisés dans des chambres d'hôtel individuelles. Pendant l'éclosion, aucun des travailleurs étrangers temporaires n'a été autorisé à se rendre dans la collectivité ou à interagir avec d'autres travailleurs à l'extérieur de leur emplacement géographique distinct.

Dans les logements partagés, un horaire rotatif a été institué pour l'utilisation des aires communes afin d'assurer la distanciation physique. On a également amélioré le nettoyage et la désinfection des aires communes de chaque logement, comme les cuisines et les salles de bain. De la nourriture et $d$ 'autres fournitures essentielles ont été livrées à chacun des logements pendant la période de l'éclosion. La communication avec les travailleurs s'est faite principalement par téléphone et par courriel, et une application mobile (WhatsApp Inc.) a été utilisée pour transmettre des messages aux travailleurs et vérifier quotidiennement leurs symptômes. Les fournisseurs de soins de santé ainsi que le personnel de gestion portaient de l'équipement de protection individuelle complet pendant les visites sur place. Les travailleurs avaient accès à des téléphones pour maintenir le lien avec leur famille et leurs amis, et ils ont reçu leur salaire normal pendant la période de quarantaine.

Pour que l'entreprise puisse continuer à opérer, les travailleurs asymptomatiques ont été divisés en cohortes, tant dans les logements partagés que sur les lieux de travail. Des mesures de contrôle améliorées, comme des postes portatifs de lavage des mains et des outils destinés à chacun des travailleurs, ont été mises en œuvre. L'on a également recommandé aux travailleurs étrangers temporaires de maintenir une distanciation physique et d'échelonner leurs pauses pour empêcher les regroupements. Une période de mise en quarantaine de 72 heures ou un nettoyage par pulvérisation avec une solution d'eau de Javel 10:1 a été instauré pour empêcher la transmission du SRASCoV-2 à partir des produits manipulés, comme les arbres et les plantes en pot, avant qu'ils ne soient expédiés au magasin de détail.

Une équipe d'intervention médicale a effectué quatre visites sur place pendant l'enquête. Cette équipe comprenait une infirmière praticienne, une infirmière autorisée ou un médecin. Chacune des visites avait un but précis, soit la recherche de cas, le dépistage et la surveillance des cas, ainsi que l'éducation des travailleurs et des autres employés. Pendant chacune des visites, l'équipe médicale a demandé à tous les travailleurs étrangers temporaires de remplir le questionnaire de dépistage des symptômes. L'équipe a ensuite prélevé des échantillons respiratoires et évalué la condition physique des travailleurs symptomatiques, y compris ceux qui présentaient seulement des symptômes légers (à l'exception des cas déjà identifiés). Au cours de la première phase de l'enquête, 12 autres travailleurs locaux qui avaient interagi avec les travailleurs étrangers ont également été examinés afin de connaître l'ampleur de l'éclosion parmi les employés de la pépinière. On a également demandé à ces travailleurs de pratiquer l'auto-isolement pendant 14 jours. Pendant ses visites sur place, l'équipe mobile a rendu visite aux cas isolés tant pour assurer leur bien-être que pour se conformer à la procédure de surveillance. En outre, les visites ont 
également permis de sensibiliser les travailleurs et le personnel de gestion et de mettre l'accent sur les mesures de prévention.

\section{Résultats}

\section{Enquête épidémiologique}

Le 23 mars 2020, le médecin-hygiéniste d'Interior Health a été avisé par le ministère de la Santé et le ministère de l'Agriculture d'un cas soupçonné de COVID-19 chez un travailleur étranger temporaire hospitalisé. La confirmation du laboratoire fut reçue le lendemain. La recherche des contacts de routine par la santé publique a permis d'identifier un contact qui était symptomatique et avait été jugé épidémiologiquement lié (confirmé par la suite). Le 27 mars 2020, un deuxième employé qui travaillait à la même pépinière et centre de jardinage s'est présenté à l'hôpital. On a alors confirmé qu'il avait la COVID-19. Cette personne habitait un logement distinct, sans contact déclaré avec le premier cas confirmé. Cette découverte sousentendait que la transmission de la COVID-19 pouvait être plus répandue que prévu parmi les travailleurs, ce qui a incité le médecin-hygiéniste à déclarer l'éclosion. Après la première visite de l'équipe mobile le 30 mars 2020, 17 autres cas ont été confirmés.

Au total, 26 cas de COVID-19 ont été recensés, dont 23 cas confirmés et trois cas épidémiologiquement liés. Tous les cas signalés n'ont touché que des travailleurs étrangers temporaires. Des 63 travailleurs étrangers, 31 ont été testés pendant l'enquête, ce qui a donné un taux de positivité de $74 \%$ (cas confirmés seulement) parmi ceux qui ont été testés. Les cas épidémiologiquement liés se sont révélés négatifs pour le virus du SRAS-CoV-2. Ces personnes ont toutefois été traitées comme des cas, compte tenu de leur exposition et de l'histoire des symptômes compatibles avec la COVID-19. Aucun cas n'a été décelé parmi les 12 travailleurs locaux (67\% d'hommes; âge moyen de 43 ans et âge médian de 44,5 ans), possiblement en raison des mesures de contrôle mises en œuvre dès le début par le propriétaire de l'entreprise, mesures qui ont eu pour effet de limiter l'interaction entre les travailleurs locaux et les travailleurs étrangers temporaires.

Les caractéristiques des cas sont présentées dans le tableau 1. La majorité des cas liés à l'éclosion étaient de sexe masculin (77\%), avec un âge moyen et médian de 41 ans. La répartition des cas selon l'âge et le sexe correspondait à celle de la cohorte complète des 63 travailleurs étrangers temporaires. Les symptômes se sont manifestés entre le 8 mars et le 9 avril 2020. La date de commencement des symptômes n'est pas disponible pour cinq des cas (19\%). Un cas a nécessité une hospitalisation d'une nuit en raison d'une pneumonie et tous les travailleurs se sont complètement rétablis. La figure 1 montre une courbe épidémique des cas liés à l'éclosion par épisode, entre le 8 mars et le 11 mai 2020. Les dates clés de l'enquête sont également indiquées.
Tableau 1 : Caractéristiques des cas de COVID-19 inclus dans l'enquête sur l'éclosion ( $N=26$ )

\begin{tabular}{|c|c|c|}
\hline $\begin{array}{c}\text { Caractéristiques des cas } \\
\text { de COVID-19 }\end{array}$ & Nombre de cas & $\%$ du total \\
\hline \multicolumn{3}{|l|}{ Cas } \\
\hline Confirmés & 23 & $88 \%$ \\
\hline $\begin{array}{l}\text { Confirmation du lien avec } \\
\text { l'épidémie }\end{array}$ & 3 & $12 \%$ \\
\hline Total & 26 & $100 \%$ \\
\hline \multicolumn{3}{|l|}{ Symptômes } \\
\hline $\begin{array}{l}\text { Commencement des } \\
\text { symptômes (Intervalle) }\end{array}$ & $\begin{array}{r}\text { Du } 8 \text { mars au } 9 \\
\text { avril } 2020\end{array}$ & $\begin{array}{r}\text { Du } 8 \text { mars au } 9 \\
\text { avril } 2020\end{array}$ \\
\hline Asymptomatique/non fourni & 5 & $19 \%$ \\
\hline \multicolumn{3}{|l|}{ Démographie } \\
\hline Hommes & 20 & $77 \%$ \\
\hline Femmes & 6 & $23 \%$ \\
\hline \multicolumn{3}{|l|}{ Groupe d'âge (années) } \\
\hline Moins de 35 ans & 5 & $19 \%$ \\
\hline 35-39 & 5 & $19 \%$ \\
\hline $40-44$ & 8 & $31 \%$ \\
\hline 45 ans et plus & 8 & $31 \%$ \\
\hline Hospitalisations & 1 & $4 \%$ \\
\hline
\end{tabular}

\section{Mise en œuvre des cohortes de travailleurs}

Les 63 travailleurs étrangers temporaires ont été jugés avoir possiblement été exposés à la COVID-19 et ont donc dû être isolés. Les travailleurs asymptomatiques ont été séparés en cohortes dans des logements partagés puisque l'isolement de tous les travailleurs dans des chambres individuelles avec salle de bain privée (i.e. dans des chambres d'hôtel) n'était ni faisable ni pratique. Les logements fournis par l'employeur étaient propres, bien entretenus et contenaient tous les articles essentiels, comme du savon liquide, des essuie-tout et des produits de nettoyage. Au départ, tous les travailleurs asymptomatiques devaient s'isoler dans leur chambre dans le logement partagé. Par la suite, lorsque les mesures de lutte contre l'éclosion eurent été entièrement mises en œuvre, les travailleurs asymptomatiques ont pu retourner travailler dans leurs sites antérieurs. Ils devaient cependant s'assurer de respecter la distanciation physique et continuer de rester isolés dans leur logement lorsqu'ils n'étaient pas au travail. Ils effectuaient différentes tâches dans des champs et des serres géographiquement séparés des autres pépinières et n'avaient pas de contact avec l'espace de vente au détail ouvert au public. Il faut noter que, des cinq sites d'hébergement géographiquement distincts, un seul n'a eu aucun cas de COVID-19 parmi les travailleurs (cinq travailleurs étrangers temporaires). Ce lieu d'hébergement et de travail était jugé plus isolé géographiquement que les autres, en plus d'avoir eu des interactions limitées avec les autres travailleurs avant la détection de l'éclosion. 
Figure 1 : Courbe épidémique des cas de COVID-19 par date d'épisodea, du 8 mars au 11 mai 2020 ( $N=26)$

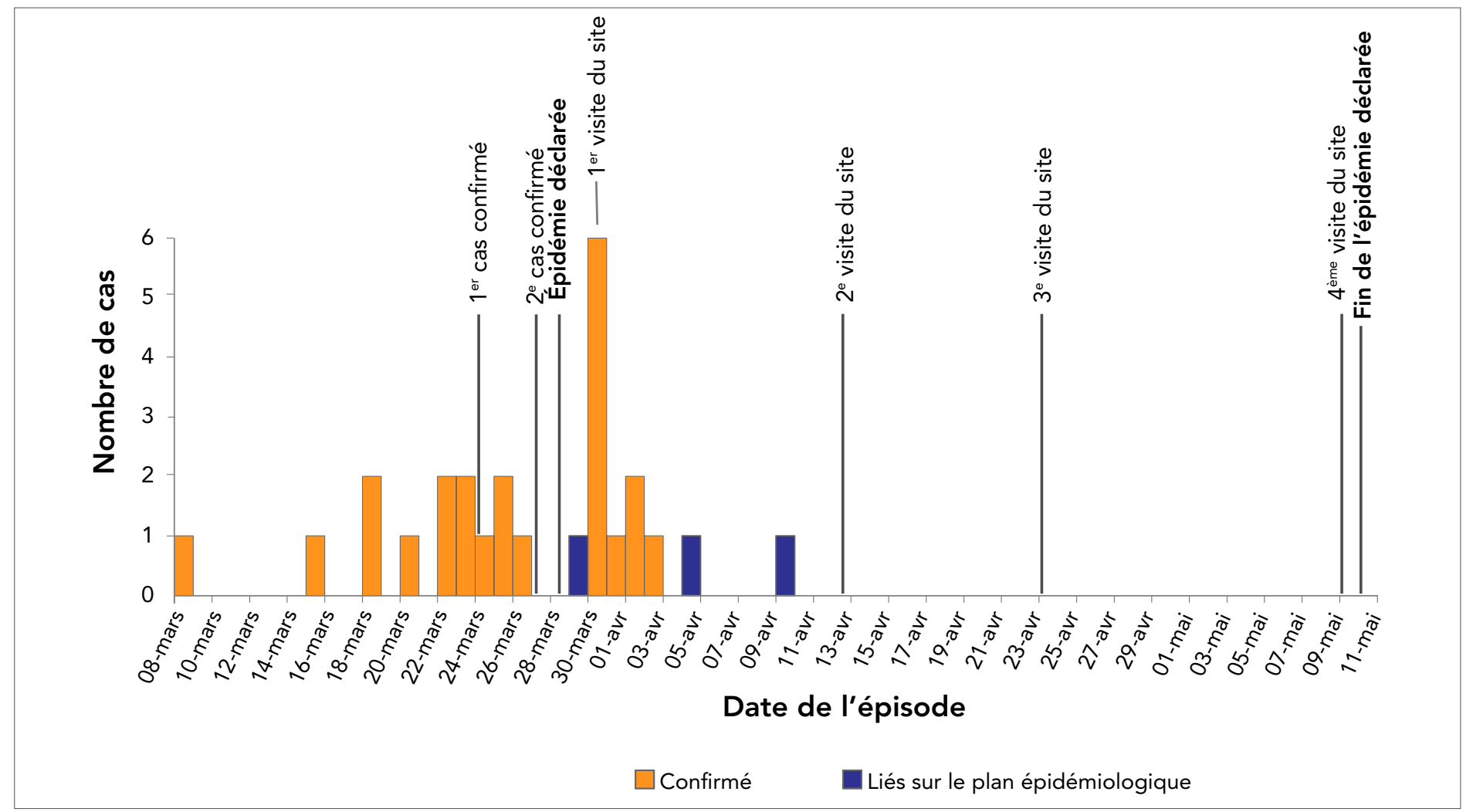

Abréviation : COVID-19, maladie à coronavirus 2019

a Date de l'épisode fondée sur l'apparition des symptômes $(n=21)$; si cette date n'est pas disponible, date de prélèvement du spécimen ( $n=5$, jusqu'au 30 mars)

\section{Discussion}

Le présent rapport résume l'éclosion de COVID-19 qui a touché un groupe de travailleurs étrangers temporaires en milieu agricole. L'éclosion a été déclarée peu de temps après que I'OMS ait jugé que la COVID-19 était une pandémie et au tout début de l'épidémie au Canada. II s'agissait de la première éclosion de COVID-19 en Colombie-Britannique et de la première éclosion de COVID-19 chez les travailleurs étrangers temporaires au Canada. Depuis, d'autres éclosions touchant les travailleurs agricoles ont été signalées (11-13).

Les travailleurs étrangers temporaires sont généralement en santé. Deux cas de COVID-19 dans des logements où habitaient différents travailleurs qui ont dû être soignés à l'hôpital ont suggéré qu'il aurait pu s'agir de la " pointe de l'iceberg " avec d'autres cas plus légers dans le groupe de travailleurs. C'est ce qui a justifié la déclaration d'une éclosion. Le dépistage subséquent de tous les travailleurs qui ont dû remplir un questionnaire de dépistage des symptômes a permis de détecter plusieurs autres cas qui n'avaient exigé aucun traitement en raison de symptômes bénins.

Au cours de l'enquête, nous avons cependant relevé un certain nombre d'obstacles aux soins chez les travailleurs étrangers temporaires. Malgré la vérification quotidienne des symptômes, on a remarqué un retard dans la déclaration des symptômes chez certaines personnes, ce qui peut être dû à des obstacles linguistiques et à la littératie en santé, mais aussi à la crainte du manque de travail, d'une perte de salaire et d'un manque de couverture des soins de santé. L'accès à des fournisseurs de services multilingues a été un facteur important qui a permis de fournir des soins appropriés et culturellement sécuritaires. Des fournisseurs de services hispanophones ont été inclus dans chacune des visites de sensibilisation, ce qui a minimisé la nécessité pour le personnel fourni par l'employeur de traduire les documents et a peut-être dissuadé les travailleurs de déclarer l'ensemble de leurs préoccupations en matière de santé. Les travailleurs étrangers temporaires avaient aussi accès à un médecin local venu du même pays qu'eux, et l'accès aux services de télésanté avec ce médecin a été facilité par les changements apportés à la facturation (14) en raison de la pandémie de COVID-19.

Les travailleurs étrangers temporaires touchés par cette éclosion sont arrivés au Canada entre le 16 janvier et le 12 mars 2020, soit juste avant les ordonnances de quarantaine provinciales et fédérales émises pour les voyageurs internationaux. Le 17 mars, l'agent de santé publique de la Colombie-Britannique a adopté une ordonnance obligeant les voyageurs internationaux qui revenaient en Colombie-Britannique ou qui y arrivaient à s'isoler pendant 14 jours (15). Une semaine plus tard, le 24 mars, le gouvernement fédéral promulguait une ordonnance de quarantaine obligatoire semblable pour les voyageurs qui 
revenaient au pays (16). Au moment de la rédaction du présent rapport (juillet 2020), tous les travailleurs étrangers temporaires qui entrent en Colombie-Britannique doivent s'isoler dans un logement géré par le gouvernement pendant 14 jours avant leur déploiement dans des fermes. Pendant cette période, les employeurs sont responsables de payer aux travailleurs un minimum de 30 heures par semaine. Du financement provincial est disponible pour les coûts d'hébergement et autres frais connexes. Les travailleurs font également l'objet d'un contrôle avant qu'ils ne quittent leur pays d'origine, ainsi qu'à leur arrivée au Canada (17). En outre, des lignes directrices nationales ont été élaborées pour aider les employeurs de travailleurs étrangers temporaires à comprendre leur rôle dans la protection de la santé et de la sécurité de leurs employés dans le contexte de la COVID-19 (18).

\section{Forces et faiblesses}

Les points forts de cette enquête incluent la collaboration entre l'entreprise touchée et les responsables de la santé pendant toute la durée de l'enquête, l'intervention coordonnée à laquelle ont pris part des intervenants internes et externes, et l'occurrence de cette éclosion dans une cohorte bien définie de travailleurs. La direction de la pépinière a été proactive en ce sens qu'elle a mis en place des mesures de prévention et de détection précoce des cas dans la période précédant la déclaration de l'éclosion. L'employeur a également versé les salaires et remis des fournitures essentielles aux travailleurs pendant toute la période d'isolement, ainsi que des locaux d'hébergement uniques, au besoin, pour les travailleurs symptomatiques. L'une des limites de cette enquête est liée au fait que nous n'avions pas testé l'ensemble des 63 travailleurs étrangers temporaires pour le SRAS-CoV-2. Nous n'avons testé que ceux qui présentaient des symptômes et un petit nombre d'autres travailleurs qui, selon nous, présentaient le risque d'infection le plus élevé compte tenu de leur exposition potentielle à un cas confirmé. Au moment de l'éclosion, I'incidence de la COVID-19 augmentait rapidement en Colombie-Britannique et on s'inquiétait de la possibilité d'une pénurie d'écouvillons nasopharyngés. Il est probable que des tests de dépistage effectués de façon plus globale parmi les travailleurs étrangers auraient permis de déceler d'autres cas. L'analyse des facteurs de risque était également limitée dans cette enquête. Lorsque l'éclosion a été détectée, une importante transmission s'était déjà produite au sein de la cohorte des travailleurs étrangers temporaires. Les logements et les groupes de travail ont également été réaménagés pendant l'enquête afin de réduire au minimum l'exposition et la transmission. Par conséquent, nous n'avons pas été en mesure de déterminer les endroits particuliers qui ont joué le rôle de facteurs de risques et ainsi contribué à l'infection symptomatique. Nous avons également été limités dans notre capacité à déterminer l'origine de l'infection qui s'est ensuite propagée dans le groupe. Il est possible que le séquençage du génome entier fournisse des renseignements supplémentaires sur le schéma de transmission de la maladie lors d'une phase future de l'analyse de l'éclosion.

\section{Conclusion}

Les travailleurs étrangers temporaires ont été exposés à des risques uniques pendant la pandémie de COVID-19, comme le démontre cette éclosion, qui s'est produite dans un milieu agricole en Colombie-Britannique. La transmission de la COVID-19 a été confirmée par l'identification et la déclaration rapides d'une éclosion et le dépistage répété des symptômes, suivis de tests de dépistage ciblés. La mise en quarantaine immédiate des travailleurs touchés, le suivi complet des cas et la recherche des contacts, ainsi que la mobilisation d'une équipe mobile ont été des stratégies efficaces pour gérer et contrôler cette éclosion. Ces mesures ont donc été mises en œuvre et ont permis à l'entreprise de poursuivre certaines de ses activités. Des directives et des ordonnances provinciales et fédérales ont depuis été élaborées afin de réduire le risque d'éclosion dans les milieux agricoles et de protéger la santé et la sécurité des travailleurs et des Canadiens dans le contexte de la pandémie.

\section{Déclaration des auteurs}

S. C. M. - Conceptualisation

S. C. M., G. F., K. H., C. Y., A. A., J. J., A. U. L., T. A., M. R. Enquête

S. C. M., G. F. - Rédaction du projet original

S. C. M., G. F., K. H., C. Y., A. A., J. J., C. G., A. U. L., T. A., M. R. - Révision et édition

\section{Intérêts concurrents}

Aucun.

\section{Remerciements}

Nous tenons à remercier toutes les personnes au sein d'Interior Health qui ont soutenu l'enquête sur l'éclosion. Nous tenons également à remercier J. Robles de KCR Community Resources pour son soutien qui nous a permis de fournir des soins de proximité culturellement sûrs.

\section{Financement}

Ce travail a bénéficié du soutien d'Interior Health.

\section{Matériel supplémentaire}

Symptom Assessment Survey (anglais) (https://www.canada. $\mathrm{ca} /$ content/dam/phac-aspc/documents/services/reportspublications/canada-communicable-disease-report-ccdr/monthlyissue/2021-47/issue-1-january-2021/ccdrv47i01a02as-eng.pdf) 
Encuesta de Evaluación de Salud (español) (https://www. canada.ca/content/dam/phac-aspc/documents/services/reportspublications/canada-communicable-disease-report-ccdr/monthlyissue/2021-47/issue-1-january-2021/ccdrv47i01a02bs-spa.pdf)

Le contenu de l'article et les points de vue qui y sont exprimés n'engagent que les auteurs et ne correspondent pas nécessairement à ceux du gouvernement du Canada.

\section{Références}

1. Organisation mondiale de la Santé. Flambée de maladie à coronavirus 2019 (COVID-19). Genève (Suisse): OMS; 2020 (accédé 2020-06-22). https://www. who.int/fr/emergencies/diseases/novel-coronavirus2019? gclid=CjOKCQiAzsz-BRCCARIsANot FgMGSdPSkCzDnSxCa3Wu9PxXOJWaN2UcHw5PZdpAYOZ bzYahe4_6nj4aArxWEALw_wcB

2. Gouvernement du Canada. Maladie à coronavirus (COVID-19) : Restrictions, exemptions et conseils en matière de voyages. Gouvernement du Canada (modifié 2020-10-15; accédé 2020-07-05). https://www.canada.ca/ en/public-health/services/diseases/2019-novel-coronavirusinfection/latest-travel-health-advice.html

3. Government of British Columbia. List of COVID-19 Essential Services. Government of BC: June 12, 2020 (accédé 2020-06-22). https://www2.gov.bc.ca/gov/content/safety/ emergency-preparedness-response-recovery/covid19-provincial-support/essential-services-covid-19

4. Agriculture et Agroalimentaire Canada. Protection de la population canadienne et des travailleurs de la chaîne d'approvisionnement alimentaire. AAAC; 13 avril 2020 (accédé 2020-06-23). https://www.canada.ca/fr/ agriculture-agroalimentaire/nouvelles/2020/04/protection-d e-la-population-canadienne-et-des-travailleurs-de-la -chaine-dapprovisionnement-alimentaire.html

5. Gouvernement du Canada. Les populations vulnérables et la COVID-19. Gouvernement du Canada (modifié 202010-01; accédé 2020-06-22). https://www.canada.ca/fr/ sante-publique/services/publications/maladies-et-affections/ populations-vulnerables-covid-19.html

6. BC Centre for Disease Control. COVID-19 (Novel Coronavirus): Case definitions. BCCDC; 2020 (accédé 2020-06-22). http://www.bccdc.ca/health-professionals/ clinical-resources/case-definitions/covid-19(novel-coronavirus)

7. British Columbia Provincial Health Services Authority. Provincial Language Service. PHSA; 2020 (accédé 2020-0622). http://www.phsa.ca/our-services/programs-services/ provincial-language-service
8. BC Centre for Disease Control. COVID-19 Case Report Form. BCCDC (modifié 2020-10-15; accédé 2020-06-22). http://www.bccdc.ca/Documents/COVID-19_Case_Report_ Form.pdf

9. Government of British Columbia. Public Health Act (accédé 2020-06-22). https://www2.gov.bc.ca/gov/content/health/ about-bc-s-health-care-system/legislation/public-health-act

10. BC Centre for Disease Control. Public Health Management: Surveillance and guidance for public health management of COVID-19. BCCDC; 2020 (accédé 2020-06-22).

http://www.bccdc.ca/health-professionals/clinical-resources/ covid-19-care/public-health-management

11. Chatham-Kent Public Health. News Release: April 27, 2020 (accédé 2020-06-22). https://ckphu.com/wp-content/ uploads/2020/04/Outbreak-Greenhill-Produce.pdf

12. Windsor-Essex County Health Unit. News Release: May 26th Public Health Updates Related to Coronavirus (COVID-19) (accédé 2020-06-22). https://www.wechu.org/ newsroom/news-release-may-26th-public-health-updates-re lated-coronavirus-covid-19

13. Windsor-Essex County Health Unit. News Release: June 12th Public Health Updates Related to Coronavirus (COVID-19). WECHU; June 12, 2020 (accédé 2020-06-22). https://www. wechu.org/newsroom/news-release-june-12th-public-healthupdates-related-coronavirus-covid-19

14. Doctors of BC. Billing changes - COVID-19. News release: June 5, 2020 (accédé 2020-07-05). https://www.doctorsofbc. $\mathrm{ca} /$ news/covid-19-temporary-billing-changes

15. Government of British Columbia. Notice to people who have been or have likely been exposed to SARS-CoV-2 (Class). Government of BC; March 17, 2020 (accédé 2020-06-22). https://www2.gov.bc.ca/assets/ gov/health/about-bc-s-health-care-system/office-o f-the-provincial-health-officer/reports-publications/covid19-pho-class-order-travellers.pdf

16. Government of Canada. Orders in Council - Search (PC Number 2020-0175). Government of Canada; March 24, 2020 (accédé 2020-06-22). https://orders-in-council.canada. ca/attachment.php?attach=38989\&lang=en

17. Government of British Columbia. Temporary foreign farmworkers must self-isolate. Government of BC (modifié 2020-10-23; accédé 2020-07-05). https://www2.gov. bc.ca/gov/content/industry/agriculture-seafood/covid-19response/temporary-foreign-farmworkers

18. Emploi et Développement social Canada. Lignes directrices pour les employeurs de travailleurs étrangers temporaires concernant la COVID-19 (modifié 2020-04-22; accédé 202007-05). https://www.canada.ca/fr/emploi-developpementsocial/services/travailleurs-etrangers/conformite-employeurs/ lignes-directrices-lecovid.html 